\title{
The emerging mysteries of IgG4-related disease
}

\author{
Authors: Wouter Smit ${ }^{\mathrm{A}}$ and Eleanor Barnes ${ }^{\mathrm{B}}$
}

IgG4-related disease (IgG4-RD) is increasingly recognised in Western societies as a multi-system, inflammatory, fibrosing disease of unknown aetiology that typically, though not exclusively, presents in older men. The clinical manifestations are diverse and almost any organ may be affected. The cardinal histological features are a lymphoplasmacytic infiltrate, storiform fibrosis, obliterative phlebitis and an abundance of IgG4+ plasma cells in affected organs. Serum IgG4 levels are elevated in approximately $70 \%$ of patients and are a useful biomarker when present. IgG4-RD is frequently misdiagnosed as malignancy. Making the correct diagnosis is important as the disease is usually steroid responsive, although relapse rates are high. Second-line immunosuppressive agents and B-cell depletion therapy have also been used in retreatment strategies. Recent data suggests that the disease is associated with both progressive organ failure and malignancy. The biological mechanisms driving IgG4-RD remain unclear but this is currently an area of intense scientific investigation. Broadly, IgG4+B cells are thought to exhibit a regulatory phenotype, but it is not known if these are pathogenic or simply represent a bystander effect. Extending our understanding of the role of IgG4 immunoglobulins in health and disease, the assessment of B and T cell immune phenotype, and large genetic studies of IgG4-RD may enhance our understanding of disease pathogenesis. Ultimately it may be that there is not a single, simple unifying aetiology and so careful stratification of disease by clinical phenotype will be required in multi-centre prospective clinical cohorts. These cohorts will also be essential for the study of treatment outcomes with novel therapies.

KEYWORDS: IgG4 related-disease, IgG4-RD, autoimmune pancreatitis, IgG4-related sclerosing cholangitis, review

\section{Introduction}

Over the past decade a new multiorgan fibroinflammatory disease characterised by IgG4-producing plasma cells in affected tissues has emerged, called IgG4-related disease

Authors: ${ }^{\text {A }}$ linician scientist, Academic Medical Center, University of Amsterdam, Amsterdam, The Netherlands; ${ }^{\text {B }}$ professor of hepatology and experimental medicine, Peter Medawar Building for Pathogen Research, Translational Gastroenterology Unit, and Oxford NIHR BRC, Oxford, UK
(IgG4-RD). From a clinical perspective, IgG4-RD presents as a remarkable array of different clinical manifestations, but with distinct pathogenic features. Since its recognition in 2003, the clinical literature on IgG4-RD has expanded exponentially and with it our understanding of the disease phenotype. However, we still lack fundamental insight into disease aetiology and immunopathogenesis. In this article we will discuss the history of the disease, the clinical manifestations, therapy and disease course. However, a focus of this review will be a description of our current understanding of disease pathogenesis and aetiology and an outline of the clinical and scientific areas that we believe warrant further investigation.

\section{A brief history}

A detailed description of pancreatic IgG4-RD dates back to 1961 with the first case of a specific form of pancreatitis ('chronic inflammatory sclerosis of the pancreas') with hypergammaglobulineamia and local lymphoplasmacytic cell infiltrates in the pancreatic lesion. ${ }^{1}$ Even at this early stage it was thought that the disease was autoimmune in nature, although it was not until 1995 that the disease was renamed autoimmune pancreatitis (AIP). ${ }^{2}$ In 2001, it was observed that serum levels of IgG4 were elevated in AIP and IgG4 became a diagnostic biomarker for disease. ${ }^{3}$ Finally in 2003, after multiple extrapancreatic lesions in AIP patients were described, ${ }^{4}$ it became evident that the immune process responsible for AIP was not just confined to the pancreas, but that this was a systemic disease with the potential to affect many organs. During the following decade, clinicians aligned to multiple medical specialties would encounter organ manifestations of IgG4$\mathrm{RD}$. As such, the disease spectrum as we understand it today consists of clinical manifestations previously described (eg Riedel's thyreoiditis and Kuttner's tumour), those that have been relabelled (ie AIP and sclerosing cholangitis) and those that are still being discovered (eg testicular IgG4-RD). The clear unifying feature is the strikingly similar histopathological features irrespective of the organ involved.

\section{Defining the diagnosis}

Correctly diagnosing IgG4-RD is critical for patient management, natural history studies and scientific studies of disease pathogenesis. Elevated serum concentrations of IgG4 are seen in more than $70 \%$ of patients with IgG4-RD and are a very useful biomarker of disease, although this alone is not diagnostic. ${ }^{5,6}$ The well-accepted Japanese consensus criteria (the HISORt criteria) incorporate IgG4 serum levels with a 
combination of clinical, radiological, and histopathological features that define the diagnosis of AIP. The recent Japanese Comprehensive Clinical Diagnostic criteria for IgG4-RD can be used to diagnose IgG4-RD regardless of the organ involved. ${ }^{7}$ The cardinal histological features are:

$>$ a dense lymphoplasmacytic infiltrate with storiform (whirlshaped) fibrosis

> obliterative phlebitis with a variable presence of eosinophils

$>$ an abundance of IgG4-bearing plasma cells (diagnostic threshold of IgG4+/total IgG+ plasma cells ratio > 40\% in the same tissue).

For some specific clinical manifestations such as IgG4associated cholangitis (IAC), a diagnostic algorithm has been developed to aid diagnosis that incorporates the ratio of IgG4 to IgG1 in serum. ${ }^{6,8}$

\section{Clinical picture}

The clinical manifestations of IgG4-RD depend on the pattern of organ involvement and the severity of the disease activity. Typically, the affected patient is a middle-aged or elderly man with symptoms related to swelling of the target organ, such as obstructive jaundice (AIP/IAC), hydronephrosis (retroperitoneal fibrosis) or painless bilateral swollen lacrimal and/or salivary glands (sialadenitis or Mikulicz's disease). Constitutional symptoms are usually absent or subtle if present, even in the setting of multiorgan involvement. Over time, and without therapy, persistent inflammation in affected organs will often lead to fibrosis and permanent organ dysfunction or failure. Distinguishing IgG4-RD from cancer may prove difficult, since imaging features are not diagnostic. This may lead to surgical intervention and organ resection for a mistaken diagnosis of malignancy. ${ }^{9}$ A significant proportion (up to $40 \%$ ) of patients with IgG4-RD have a longstanding history of allergic disease or atopic features, ${ }^{10,11}$ raising interesting questions as to the role of allergy in disease pathogenesis. Our data, and that of others, ${ }^{12}$ suggests IgG4-RD may be associated with the development of end stage organ failure, liver cirrhosis and malignancy - both in the pancreas and also at more distal anatomical sites. Large prospective studies of natural history are required to realise the full extent of disease progression and the risks of developing malignancy.

\section{Treatment}

Although not based on comparative clinical trials, the first line of treatment in the USA and Europe currently typically involves an initial course of 3-6 months of corticosteroid therapy beginning at 20-30 mg daily. The response to steroids (through imaging and biochemical assessment) may be assessed 6-8 weeks later. At 3-6 months, steroid therapy is usually tapered gradually and then stopped. Remission rates are high (99.6\% in one multinational analysis of 978 patients with IgG4-related pancreatitis). ${ }^{13}$ Although initially effective, longterm steroid therapy renders these patients at risk of serious side-effects. Moreover, relapse after cessation of steroids is common. At the University of Oxford and University College London, we have observed relapse rates of approximately $50 \%$ in patients with AIP after stopping steroid therapy, with relapse more commonly observed in patients with hepatobiliary disease. In cases of steroid dependency or refractory disease, contraindications or intolerable side effect, immunomodulators (mycophenolate mofetil, azathioprine and 9-mercaptopurin) may be used in addition or as an alternative to steroids. B cell depletion with rituximab, an anti-CD20 antibody, has shown promising early results. ${ }^{14}$

\section{Aetiology and pathogenesis \\ IgG4-antibodies - pathogenic or bystander effect?}

Affected organs in IgG4-RD contain a diffuse inflammatory infiltrate that consists of polyclonal $\mathrm{T}$ (mostly $\mathrm{T}$ helper cells) and B lymphocytes (comprising all antibody subclasses but predominately IgG4), plasma cells, and sometimes eosinophils. Although infiltrating IgG4+ B cells are an important feature of disease, IgG4+ B cell infiltration may be seen in other inflammatory conditions. ${ }^{15}$ The fundamental question of whether the IgG4+ B cells are pathologically involved, driving inflammation and contributing to tissue damage and consequent fibrosis, is currently not resolved. Alternative explanations are that the infiltrating B cells merely reflect an inert 'bystander effect' of the inflammatory process, or that this cell population is recruited into an inflamed organ to downregulate the inflammatory process.

Further understanding the biology of IgG4+ B cells might give insight into the role of these cells in disease. IgG4 is found in low abundance in the serum of healthy people in comparison to other IgG subclasses, and has some unique properties. For example, the IgG4 antibody variable region is bispecific and can undergo 'fab-arm exchange'. In effect this means that IgG4 is functionally monovalent and unable to form large immune complexes. Furthermore, IgG4 is unable to activate the classical complement pathway, shows reduced binding to classical Fc gamma receptors (ie Fc $\gamma$ RI, Fc $\gamma$ RIIa and Fc $\gamma$ RIIIa), ${ }^{16}$ and may interfere with complement-activating and immuneprecipitating activities of IgG1. ${ }^{16}$ Together this suggests that IgG4 has predominantly regulatory, rather than stimulatory, immune functions. Until recently, the capacity to investigate the phenotype or ex-vivo function of IgG4+ B cells in either health or disease has been limited by the lack of IgG4-specific antibodies for FACS analysis. The recent development at the Sanquin Blood Supply, Division of Research in Amsterdam, of an IgG4 specific antibody for FACS therefore represents a significant step forward in the field. ${ }^{17}$ The recent isolation of IgG4+ B cells with a memory phenotype (IgD-CD27+) in healthy individuals has shown that these cells express less complement and Fc receptors (CD21 and CD23) than IgG1+ cells which might decrease the threshold for B cell activation. ${ }^{17}$ These very recent observations highlight the fact there is much yet to understand about IgG4+ B cells in both health and disease.

\section{Antigens in IgG4-RD}

IgG4 normally accounts for $3-6 \%$ of total serum IgG, but can represent up to $80 \%$ under certain situations of chronic or repetitive antigenic exposure, representing the development of humoral tolerance. ${ }^{18}$ In certain groups, such as experienced bee keepers (who develop anti-PLA IgG4) and patients who receive hyposensitisation therapy with allergen (ie housedust mite and grass pollen) or specific immune therapy and 
treatment with certain proteins (ie factor VIII, adalimumab), remarkable elevations of serum IgG4 can be seen. ${ }^{18}$ These antiinflammatory or tolerance-inducing mechanisms of IgG4 argue for a possible regulatory function in IgG4-RD. However, these observations also show that specific exogenous antigens are able to drive IgG4+ B cell responses. Furthermore, pathogenic IgG4+ autoantibodies including IgG4 against desmoglein-1 in pemphigus vulgaris or MuSK protein in myasthenia gravis are well described.

The role of specific antigens, either endogenous or exogenous, in driving IgG4-RD, is currently debated and under investigation. The observation of an HLA-class II association with IgG4$\mathrm{RD}$ in Asian populations implicates the antigen presentation pathway in disease. ${ }^{19}$ It is clear that IgG4+ B cells develop after B cells undergo class switching, and so the IgG4+ B cells that are predominant in IgG4-RD must have been exposed to antigen. However that is not to say a specific antigen is implicated in either the disease or in the generation of an IgG4+ B cell response. It is equally possible that IgG4+ B cells of multiple specificities are amplified in response to an inflammatory environment. One antigen that has been implicated in pancreatic IgG4-RD (AIP) is Helicobacter pylori, following the observation that antibodies against a Helicobacter surface protein could be found in $94 \%$ of an Italian cohort with AIP. ${ }^{20}$ However, these results have not yet been confirmed elsewhere, and an attempt to verify this in the UK cohort has failed to support this observation (unpublished data). Hence, a role for H pylori antigen in IgG4-RD remains contentious.

One way to assess the role of antigen specificity is through the assessment of the B cell receptor repertoire. It has been shown that a subset of IgG4+ B cell clones may dominate in IgG4$\mathrm{RD}$, and that the same clones can be identified in both the

\section{Key learning points}

IgG4-RD is increasingly recognised in western populations.

The disease typically affects older males.

> Although the disease often presents to gastroenterologists with pancreatic and hepatobiliary disease, many others organs may be affected.

$>$ Serum IgG4 levels are elevated in only $70 \%$ of patients with IgG4-RD.

> Histology is characterised by a storiform fibrosis, a plasmalymphocytic infiltrate and IgG4 producing B cells.

The disease may respond to 3-6 months of corticosteroid therapy but relapse is common.

IgG4-RD is not necessarily a benign condition and may be associated with progressive organ failure and malignancy.

Further studies of natural history, genetic analysis and treatment are required in large, carefully phenotyped patient cohorts.

In the medium term, the research agenda is likely to focus on the assessment of $\mathrm{B}$ and $\mathrm{T}$ cell phenotype and function in disease, the biology of IgG4+ B cells in both health and disease, and the assessment of the B cell receptor repertoire in disease. blood and in diseased organs in IgG4-RD patients. ${ }^{21}$ A similar study found oligoclonal expansion of the plasmablast pool in patients with active disease. ${ }^{22}$ However, no clones were found that were identical between infected individuals, and further data from our group (manuscript in submission) shows that the IgG4+ B cell response in IgG4-RD is polyclonal with the capacity to target multiple exogenous antigens. Collectively, these results suggest that an expanded population of clonally partially restricted B cells or plasmablasts may be found in IgG4-RD but the role of these in pathogenesis remains unclear. Furthermore, the spectrum of clonality of $\operatorname{IgG} 4+\mathrm{B}$ cells in healthy individuals is largely unexplored. Further analysis of $B$ and $T$ cell clonality in IgG4-RD patients carefully classified by disease phenotype, in other conditions where IgG4+ B cells are increased, and in health may increase our understanding of IgG4-RD pathogenesis and antigenic drivers of disease.

\section{Clues to pathogenesis in response to therapy}

Assessment of the immune response to effective therapies, or to the immune response during disease relapse, may give important clues into IgG4-RD pathogenesis. Tracking immune responses in patients receiving $\mathrm{CD} 20+\mathrm{B}$ cell depletion therapy with rituximab may be particularly informative. During rituximab therapy, there is a rapid clinical response with a decline of serum IgG4 levels, while other IgG subclasses decrease only slightly. ${ }^{14}$ This data suggests that rituximab is depleting CD20+ IgG4+ B cells with a short half-life and that these cells may have a pathogenic role, rather than through a direct effect on plasma cells that do not express CD20. An expanded circulating IgG4+ plasmablast pool with increased expression levels of HLA-DR and B-cell maturation antigen that was lost after one month of rituximab treatment and re-emerged at the time of relapse has been described, with monoclonal immunoglobulins derived from the most abundant IgG4+ plasmablasts showing an 'autoreactive signature'. ${ }^{22}$ It is suggested that these plasmablasts could be generated de novo from naïve B cells at disease initiation and from memory B cells that have survived rituximab therapy during disease relapse. Alternatively CD20-negative bone-marrow or tissue residing plasma cells, unaffected by rituximab could contribute to relapse. These findings highlight the need to further evaluate and characterise different B cell subsets in blood and affected tissue during the course of disease, and to assess the clinical utility of plasmablasts as a biomarker for disease activity in a large patient cohort.

\section{T cells in disease}

The role of T cells in pathogenesis and the interactions with $\mathrm{B}$ cells have yet to be elucidated. T cells may contribute to the IgG4 class-switching and mediate inflammation and fibrosis. With current evidence suggesting HLA class-II associations with disease, $\mathrm{T}$ helper cell-dependent antigen recognition is likely to be involved. Furthermore, T cells could mediate humoral IgG4 responses through the secretion of cytokines that favour the development of IgG4 antibodies. It has been shown that physiological IgG4 responses, like IgE, can be controlled in vivo by Th 2 cells via induction of IL4 and IL13, directing naïve B cells to switch to IgG4 and IgE. IL10 produced by Th2 cells and Tregs also preferentially promotes class-switch 
from IgE towards IgG4. ${ }^{23,24}$ Interestingly, several studies have shown an abundant presence of Th2 cells and Th2-related cytokines in the affected organs, ${ }^{25}$ while others have shown an increase in Tregs in IgG4-RD. ${ }^{26}$ Whether these cells are causative in the disease process or are simply recruited in response to the disease process is currently unknown. IL10 and high IgG4 levels are produced in response to BAFF-dependent stimulation, implicating a role for innate immunity via Tolllike receptor and Nod-like receptor stimulation. ${ }^{27}$ Detailed analysis of $\mathrm{T}$ cell phenotype and cytokine production in diseased tissues and peripheral blood using flow cytometry and immunohistochemical analysis may shed further light on the role of $\mathrm{T}$ cells in pathogenesis.

\section{Genetic susceptibility}

Irrespective of the factors that initiate disease, genetic factors may lead to disease susceptibility. Candidate gene studies in Asian populations have shown association of HLA serotypes DRB1 ${ }^{*} 0405$ and DQB1 ${ }^{\star} 0401$, and single-nucleotide polymorphisms in non-HLA immune genes (cytotoxic T-lymphocyte-associated antigen 4 , tumor necrosis factor- $\alpha$, and Fc receptor-like). ${ }^{19,28-30}$ A genome-wide approach to identify genetic factors associated with disease is warranted, but this will require a multicentre collaborative effort to derive sufficient patient numbers to power the studies.

\section{Conclusion}

IgG4 is increasingly recognised in western countries and typically presents in older males with pancreatic and biliary pathology. Over recent years there is increasing recognition of the diverse clinical manifestations, the systemic nature of disease, and of the association with progressive organ failure and malignancy. In the medium term, future study on B cell biology and genetics may shed new light on disease pathogenesis. Underlying environmental factors that may potentially initiate disease such as allergens, infectious agents and occupational exposure will remain a focus. It may be that ultimately there is not a single unifying cause despite the common histological findings, and careful stratification of patient populations by clinical phenotype may be required to properly define both aetiology and natural history. Multicentre prospective cohort studies and clinical studies of treatment are now required to optimise therapeutic strategies, and to provide novel biomarkers of disease.

\section{Acknowledgements}

EB is supported by the MRC as a senior clinical fellow, the Oxford NHIR BRC has provided support for the patient cohort at Oxford. We would also like to thank Emma Culver (WT clinical training fellow) for support to Wouter Smit in his written and laboratory work during his time at Oxford and for her research on IgG4 with EB at Oxford.

\section{References}

1 Sarles H, Sarles JC, Muratore R and Guien C. Chronic inflammatory sclerosis of the pancreas - an autonomous pancreatic disease? Am J Dig Dis 1961;6:688-98.

2 Yoshida K, Toki F, Takeuchi T et al. Chronic pancreatitis caused by an autoimmune abnormality. Proposal of the concept of autoimmune pancreatitis. Dig Dis Sci 1995;40:1561-8.
3 Hamano H, Kawa S, Horiuchi A et al. High serum IgG4 concentrations in patients with sclerosing pancreatitis. $N$ Engl J Med 2001;344:732-8.

4 Kamisawa T, Funata N, Hayashi Y et al. Close relationship between autoimmune pancreatitis and multifocal fibrosclerosis. Gut 2003;52:683-7.

5 Sadler R, Chapman RW, Simpson D et al. The diagnostic significance of serum IgG4 levels in patients with autoimmune pancreatitis: a UK study. Eur J Gastroenterol Hepatol 2011;23:139-45.

6 Kawa S, Ito T, Watanabe T et al. The utility of serum IgG4 concentrations as a biomarker. Int J Rheumatol 2012;2012:198314

7 Umehara H, Okazaki K, Masaki Y et al. Comprehensive diagnostic criteria for IgG4-related disease (IgG4-RD), 2011. Mod Rheumatol 2012;22:21-30.

8 Boonstra K, Culver EL, de Buy Wenniger LM et al. Serum immunoglobulin G4 and immunoglobulin G1 for distinguishing immunoglobulin G4-associated cholangitis from primary sclerosing cholangitis. Hepatology 2013;59;1954-63.

9 Tripathi M, Mudhar HS, Start R and Fernando MS. IgG4-related disease - a modern mimic of malignancy. Diagnostic Histopathol 2010;16:484-5.

10 Kamisawa T, Anjiki H, Egawa N and Kubota N. Allergic manifestations in autoimmune pancreatitis. Eur J Gastroenterol Hepatol 2009;21:1136-9.

11 Della Torre E, Mattoo H, Mahajan VS et al. Prevalence of atopy, eosinophilia, and IgE elevation in IgG4-related disease. Allergy 2014;69:269-72.

12 Yamamoto M, Takahashi H, Tabeya $\mathrm{T}$ et al. Risk of malignancies in IgG4-related disease. Mod Rheumatol 2012;22:414-8.

13 Hart P, Kamisawa T, Brugge WR et al. Long-term outcomes of autoimmune pancreatitis: a multicentre, international analysis. Gut 2013;62;1771-6.

14 Khosroshahi A, Bloch DB, Deshpande V and Stone JH. Rituximab therapy leads to rapid decline of serum IgG4 levels and prompt clinical improvement in IgG4-related systemic disease. Arthritis Rheum 2010;62:1755-62.

15 Raina A, Yadav D, Regueiro M et al. Mucosal IgG4 cell infiltration in ulcerative colitis is linked to disease activity and primary sclerosing cholangitis. Inflamm Bowel Dis 2013;19;1232-7.

16 Van der Zee JS, van Swieten P and Aalberse RC. Inhibition of complement activation by IgG4 antibodies. Clin Exp Immunol 1986;64:415-22.

17 Lighaam LC, Vermeulen E, Bleker TD et al. Phenotypic differences between $\operatorname{IgG4}(+)$ and $\operatorname{IgG1}(+)$ B cells point to distinct regulation of the IgG4 response. J Allergy Clin Immunol 2014;133:267-70.

18 Aalberse RC, Stapel SO, Schuurman J and Rispens T. Immunoglobulin G4: an odd antibody. Clin Exp Allergy 2009;39:469-77.

19 Kawa S, Ota M, Yoshizawa K et al. HLA DRB10405-DQB10401 haplotype is associated with autoimmune pancreatitis in the Japanese population. Gastroenterology 2002;122:1264-9.

20 Frulloni L, Lunardi C, Simone R et al. Identification of a novel antibody associated with autoimmune pancreatitis. N Engl J Med 2009;361:2135-42.

21 De Buy Wenniger LJM, Doorenspleet ME, Klarenbeek PL et al. IgG4+ clones identified by next-generation sequencing dominate the B-cell receptor repertoire in IgG4-associated cholangitis. Hepatology 2013;57:2390-8.

22 Mattoo H, Mahajan VS, Della-Torre E et al. De novo oligoclonal expansions of circulating plasmablasts in active and relapsing IgG4related disease. J Allergy Clin Immunol 2014;134:679-87.

23 Punnonen J, Aversa G, Cocks BG et al. Interleukin 4-independent IgG4 and IgE synthesis and CD23 expression by human. Immunology 1993;90:3730-4.

24 Jeannin P, Lecoanet S, Delneste Y, Gauchat JF and Bonnefoy JY. IgE versus IgG4 production can be differentially regulated by IL-10. J Immunol 1998;160:3555-61. 
25 Zen Y, Fujii T, Harada K et al. Th2 and regulatory immune reactions are increased in immunoglobin G4-related sclerosing pancreatitis and cholangitis. Hepatology 2007;45:1538-46.

26 Kusuda T, Uchida K and Miyoshi H. Involvement of inducible costimulator - and interleukin 10 - positive regulatory $\mathrm{T}$ cells in the development of IgG4-related autoimmune pancreatitis. Pancreas 2011;40:1120-30

27 Akitake R, Watanabe T, Zaima C et al. Possible involvement of T helper type 2 responses to Toll-like receptor ligands in IgG4-related sclerosing disease. Gut 2010;59:542-5.

28 Chang MC, Chang YT, Tien YW et al. T-cell regulatory gene CTLA-4 polymorphism/haplotype association with autoimmune pancreatitis. Clin Chem 2007;53:1700-5.
29 Umemura T, Ota M, Hamano $\mathrm{H}$ et al. Association of autoimmune pancreatitis with cytotoxic T-lymphocyte antigen 4 gene polymorphisms in Japanese patients. Am J Gastroenterol 2008;103:588-94.

30 Umemura T, Ota M, Hamano $\mathrm{H}$ et al. Genetic association of Fc receptor-like 3 polymorphisms with autoimmune pancreatitis in Japanese patients. Gut 2006;55:1367-8.

Address for correspondence: Dr E Barnes, Peter Medawar Building for Pathogen Research, South Parks Road, Oxford, OX1 3SY, UK.

Email: ellie.barnes@ndm.ox.ac.uk; wouter.vriend@gmail.com 